within $0.04 \AA]$. The dihedral angle between the equatorial $\mathrm{CuO}_{4}$ plane and the chelate plane is $158.3^{\circ}$. Bond lengths and bond angles in the ligand adopt values typical for acetylacetonates $[\mathrm{C}-\mathrm{C}(\mathrm{C})=\mathrm{O}$ fragment $]$ (Lingafelter \& Braun, 1966) and phosphonic acid derivatives $\left[\mathrm{O}=\mathrm{P}(\mathrm{O})_{2}-\mathrm{C}\right.$ fragment] (Naumov \& Vilkov, 1986). The angles in the $\mathrm{P}(\mathrm{C}) \mathrm{O}_{3}$ tetrahedron vary from $98.0(6)[\mathrm{O}(3 a)-\mathrm{P}-\mathrm{O}(3 b)]$ to $116.2(5)^{\circ}[\mathrm{O}(2)$ $\mathrm{P}-\mathrm{O}(3 a)]$. The conformation of both propoxy groups is different and follows the requirements of molecular packing. Torsion angles $\mathrm{P}-\mathrm{O}-\mathrm{C}-\mathrm{C}[\mathrm{O}-\mathrm{C}-\mathrm{C}-\mathrm{C}]$ are $158(2),-172(1)\left[-20(3),-180(2)^{\circ}\right]$ for branches $a$ and $b$ respectively.

The cyano group with a $\mathrm{C}(4)-\mathrm{N}$ bond length of 1.15 (1) $\AA$ has triple-bond character. Consideration of the relatively short $\mathrm{Cu} \cdots \mathrm{N}$ distance, and the geometry of the cyano groups within the copper coordination sphere [angle $\mathrm{Cu} \cdots \mathrm{N} \equiv \mathrm{C} \quad\left(-x, \frac{1}{2}+y, \frac{1}{2}-z\right)=$ $\left.149.8(9)^{\circ}\right]$, indicates that $\pi$-electron delocalization of $\mathrm{C} \equiv \mathrm{N}$ is effectively compensated by $\sigma$-electron transfer from $\mathrm{Cu}$ to $\mathrm{N}$. In this structure the ligand is tridentate and performs a chelate-bridging role (Shkolnikova \& Poray-Koshits, 1982).

\section{References}

Enraf-Nonius (1985). Structure Determination Package. SDP/ PDP V3.0 User's Guide. Enraf-Nonius, Delft, The Netherlands.

Lingafelter, E. C. \& BRAun, R. L. (1966). J. Am. Chem. Soc. 88, 2951-2956.

Naumov, V. A. \& Vזrkov, L. V. (1986). Molecular Structures of Phosphorus-Organic Compounds, p. 317. Moscow: Science.

Petrov, G. (1972). Dissertation, p. 214. Univ. Sofia, Bulgaria.

Petrov, G. \& KirILov, M. (1968). Monatsh. Chem. 99, 19231931.

Procter, I. M., Hathaway, B. J. \& Nichols, P. (1968). J. Chem. Soc. $A$, pp. 1678-1684.

Shrolnikova, L. M. \& Poray-Koshits, M. A. (1982). Itogi Nauki Tekh. Kristallokhim. 16, 117-231.

Tomlinson, A. A. G., Hathaway, B. J., Billing, D. E. \& Nichols, P. (1969). J. Chem. Soc. A, pp. 65-71.

Weiss, E., Kopf, J., Gardein, T., Corbelin, S., Schuman, U., Kirilov, M. \& Petrov, G. (1985). Chem. Ber. 118, 3529-3534.

Acta Cryst. (1988). C44, 628-631

\title{
Structures of Chloro(glycinato)(1,10-phenanthroline)copper(II) Monohydrate (I) and Aqua(1,10-phenanthroline)(L-phenylalaninato)copper(II) Nitrate Monohydrate (II)
}

\author{
By X. Solans \\ Dpto Cristalografía, Mineralogía y Depósitos Minerales, Universidad de Barcelona, Martí y Franqués, \\ 08020 Barcelona, Spain \\ L. Ruiz-Ramírez, A. MARTINeZ AND L. GasQue \\ Dpto Química Inorgánica, Facultad de Química, Universidad Nacional Autónoma de México, CP, \\ 04510 Mexico DF
}

AND J. L. BRIANSÓ

Dpto Geologia, Universidad Autónoma de Barcelona, 08193 Bellaterra, Spain

(Received 6 May 1987; accepted 9 December 1987)

\begin{abstract}
I) $\left[\mathrm{CuCl}\left(\mathrm{C}_{2} \mathrm{H}_{4} \mathrm{NO}_{2}\right)\left(\mathrm{C}_{12} \mathrm{H}_{8} \mathrm{~N}_{2}\right)\right] \cdot \mathrm{H}_{2} \mathrm{O}, M_{r}$ $=371.28$, orthorhombic, $P 22_{1} 2_{1}, a=6.795$ (3), $b$ $=12.496(4), c=17.273(5) \AA, V=1467(1) \AA^{3}, D_{x}=$ $1.680 \mathrm{Mg} \mathrm{m}^{-3}, \quad Z=4, \quad F(000)=756, \quad \lambda($ Mo $K \alpha)=$ $0.71069 \AA, \mu(\mathrm{Mo} K \alpha)=1.742 \mathrm{~mm}^{-1}$. Room temperature. Final $R=0.046$ for 1302 unique observed reflections. (II) $\left[\mathrm{Cu}\left(\mathrm{C}_{9} \mathrm{H}_{10} \mathrm{NO}_{2}\right)\left(\mathrm{C}_{12} \mathrm{H}_{8} \mathrm{~N}_{2}\right)\left(\mathrm{H}_{2} \mathrm{O}\right)\right] \mathrm{NO}_{3}$.- $^{-}$ $\mathrm{H}_{2} \mathrm{O}, M_{r}=505.98$, monoclinic, $P 2_{1}, a=5.782$ (2), $b=20.700$ (6), $c=9.355$ (3) $\AA, \beta=97.58(2)^{\circ}, V=$ $1110(1) \AA^{3}, \quad D_{x}=1.514 \mathrm{Mg} \mathrm{m}^{-3}, \quad Z=2, \quad F(000)=$ 522, $\quad \lambda(\operatorname{Mo} K \alpha)=0.71069 \AA, \quad \mu($ Mo $K \alpha)=$ $1.076 \mathrm{~mm}^{-1}$. Room temperature. Final $R=0.069$ for 1929 unique observed reflections. The $\mathrm{Cu}$ ion displays
\end{abstract}

0108-2701/88/040628-04\$03.00 distorted square-pyramidal coordination in both (I) and (II), with the chlorine atom (I) or the water molecule (II) in the apical position. The $\mathrm{Cu}-\mathrm{N}$ bond lengths alter according to the electronegative character of the trans atom. The conformations of the five-membered chelate rings appear to depend on $\mathbf{H}$ bonding and van der Waals interactions.

Introduction. Interest in mixed-ligand chelate complexes has been clearly established in the last few years (Griesser \& Sigel, 1970). A series of compounds with formula $M(\mathrm{~N}-\mathrm{N})(\mathrm{O}-\mathrm{N}) \quad(M=\mathrm{Cu}, \quad \mathrm{N}-\mathrm{N}=1,10-$ phenanthroline, bipyridine, substituted 1,10-phenan(c) 1988 International Union of Crystallography 
throline or bipyridine, and $\mathrm{O}-\mathrm{N}=$ amino acid) has been synthesized and characterized, with stability constants determined potentiometrically (RuizRamirez, Martínez \& Gasque, 1988). The crystal structures of two compounds of this series, (I) and (II), are presented here with a discussion of the effect of the amino-acid ligands on the environment of the metal ion.

Experimental. A similar process was used for data collection in both structures. Dark prismatic crystals $[0.1 \times 0.1 \times 0.15 \mathrm{~mm}$ for (I) and $0.1 \times 0.1 \times 0.2 \mathrm{~mm}$ for (II)], Philips PW 1100 diffractometer, Mo $K \alpha$, graphite monochromator, cell parameters from 25 reflections $\left(4 \leq \theta \leq 12^{\circ}\right)$, $\omega$-scan technique, scan width $1^{\circ}$, scan speed $0.03^{\circ} \mathrm{s}^{-1}, 1373$ independent reflections with $\theta \leq 27^{\circ} ; 1302$ with $I \geq 2.5 \sigma(I)$ in (I); hkl range: 0 to 8 ; 0 to 14 ; and 0 to 20.2423 independent with $\theta \leq 25^{\circ} ; 1929$ with $I \geq 2.5 \sigma(I)$ in (II); $h k l$ range: -6 to $6 ; 0$ to 24 ; and 0 to 11 . Three standard reflections measured every $2 \mathrm{~h}$, no significant intensity decay, Lp correction, absorption ignored.

Both structures were solved by direct methods (MULTAN; Main, Fiske, Hull, Lessinger, Germain, Declercq \& Woolfson, 1980). Isotropic and anisotropic full-matrix least-squares refinement (SHELX76; Sheldrick, 1976), $\sum w|| F_{o}|-| F_{c}||^{2}$ minimized, $w=$ $\left[\sigma^{2}\left(F_{o}\right)+k\left|F_{o}\right|^{2}\right]^{-1}$, where $k=0.0004$ in (I) and 0.0 in (II), scattering factors and anomalous-dispersion terms from International Tables for X-ray Crystallography (1974). Ten $\mathrm{H}$ of (I) and all $\mathrm{H}$ of (II) were located from a difference synthesis and the remaining four $\mathrm{H}$ atoms of (I) were placed in computed positions, all $\mathrm{H}$ were refined with an overall isotropic temperature factor with the remaining atoms anisotropic. The four computed $\mathrm{H}$ atoms of (I) were constrained with $\mathrm{C}-\mathrm{H}=1.08 \AA$. Final $R(w R)$ were $0.046(0.052)$ for 1302 reflections in (I) and $0.069(0.095)$ for 1929 reflections in (II). Max. $\Delta / \sigma=-0.14$ in $z$ of $\mathrm{H}(\mathrm{C} 3)$ in (I) and 0.25 in $y$ of $\mathrm{H}(\mathrm{N} 8)$ in (II), max. and min. peaks in final $\Delta \rho$ map 0.4 and $-0.3 \mathrm{e} \AA^{-3}$, respectively, in both structures.

Discussion. Figs. 1 and 2 show views of the molecules with the numbering of atoms for structures (I) and (II), respectively. Final coordinates and selected bond lengths and angles are listed in Tables 1 and 2.* In both structures the $\mathrm{Cu}$ atom displays a distorted squarepyramidal coordination, with the phenanthroline and amino-acid ligands in the basal plane and $\mathrm{a} \mathrm{Cl}$ atom in (I), or a water molecule in (II), in the apical site. The average $\mathrm{Cu}-\mathrm{O}$ and $\mathrm{Cu}-\mathrm{N}$ (amino acid) bond lengths are $1.936(9)$ and $2.006(8) \AA$, while the $\mathrm{Cu}-\mathrm{N}$ -

\footnotetext{
* Lists of structure factors, anisotropic thermal parameters and $\mathrm{H}$-atom parameters have been deposited with the British Library Document Supply Centre as Supplementary Publication No. SUP 44607 (24 pp.). Copies may be obtained through The Executive Secretary, International Union of Crystallography, 5 Abbey Square, Chester CH1 2HU, England.
}

(phenanthroline) bond length alters according to the electronegative character of the trans ligand: The $\mathrm{Cu}-\mathrm{N}$ bond lengths trans to $\mathrm{O}$ are 0.033 (8) $\AA$ [average value from (I) and (II)] longer than those trans to $\mathrm{N}$. This fact was also observed in (1,10-phenanthroline)(salicylaldehydato)copper(II) nitrate (Solans, Ruiz-Ramirez, Gasque \& Briansó, 1987) where comparable values are $1.991(3)$ and 2.005 (3) $\AA$, respectively. The $\mathrm{Cu}-\mathrm{N}$ (phenanthroline) distances are shorter than those observed in the trans-bis $(1,10$ phenanthroline)copper(II) complex (Boys, Escobar \& Martínez-Carreras, 1981) [average value 2.05 (1) $\AA$ ]. The $\mathrm{Cu}-\mathrm{O}(W 1)$ bond length in (II), $2 \cdot 213(10) \AA$, is intermediate between those observed for $\mathrm{Cu}-\mathrm{ONO}_{2}$ (apical) in nitratobis(2,9-dimethyl-1,10-phenanthroline)copper(II) trichloroacetate trichloroacetic acid solvate where $\mathrm{Cu}-\mathrm{O}=2 \cdot 15$ (4) $\AA$ (Van Meerssche, Germain, Declercq \& Wilputte-Steinert, 1981) and the value of 2.402 (3) $\AA$ in (1,10-phenanthroline)(salicylaldehydato)copper(II) nitrate (Solans, RuizRamirez, Gasque \& Briansó, 1987). The $\mathrm{Cu}-\mathrm{Cl}$ (apical) bond length $[2.546(2) \AA]$ in (I) is intermediate when compared with the following values: 2.233 (1) observed in di- $\mu$-chloro-bis[chloro(1,2-cyclohexanedione dioxime- $\left.N, N^{\prime}\right)$ copper(II)] (Mégnamisi-Bélombé \& Endres, 1983); 2.463 (1) in dichloro(2,6-diacetylpyridinedioxime)copper(II) dihydrate (Nicholson, Petersen \& McCormick, 1982) and 2.565(2) in dichloro(2,2':6',2''-terpyridyl)copper(II) monohydrate

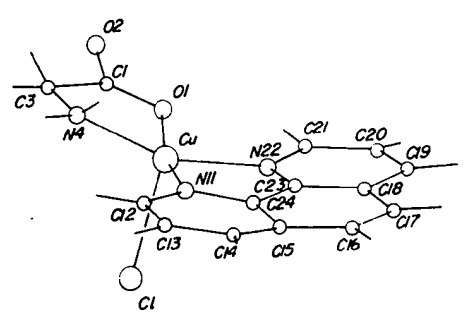

Fig. 1. View of (I) with atom numbering.

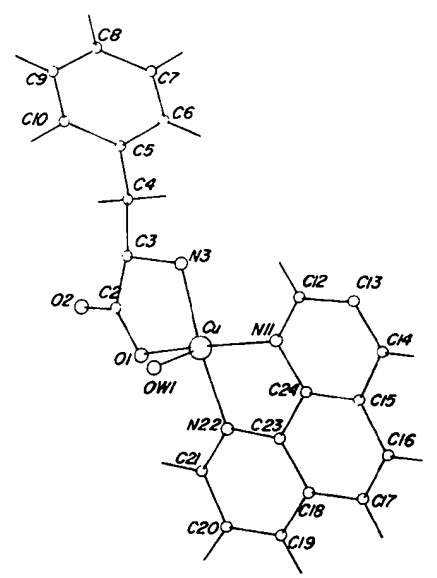

Fig. 2. View of (II) with atom numbering. 
Table 1. Final atomic coordinates $\left[\times 10^{4} ; \mathrm{Cu} \times 10^{5}\right.$ in (I)]

Table 2. Bond lengths $(\AA)$ and angles $\left(^{\circ}\right)$ with e.s.d.'s in parentheses

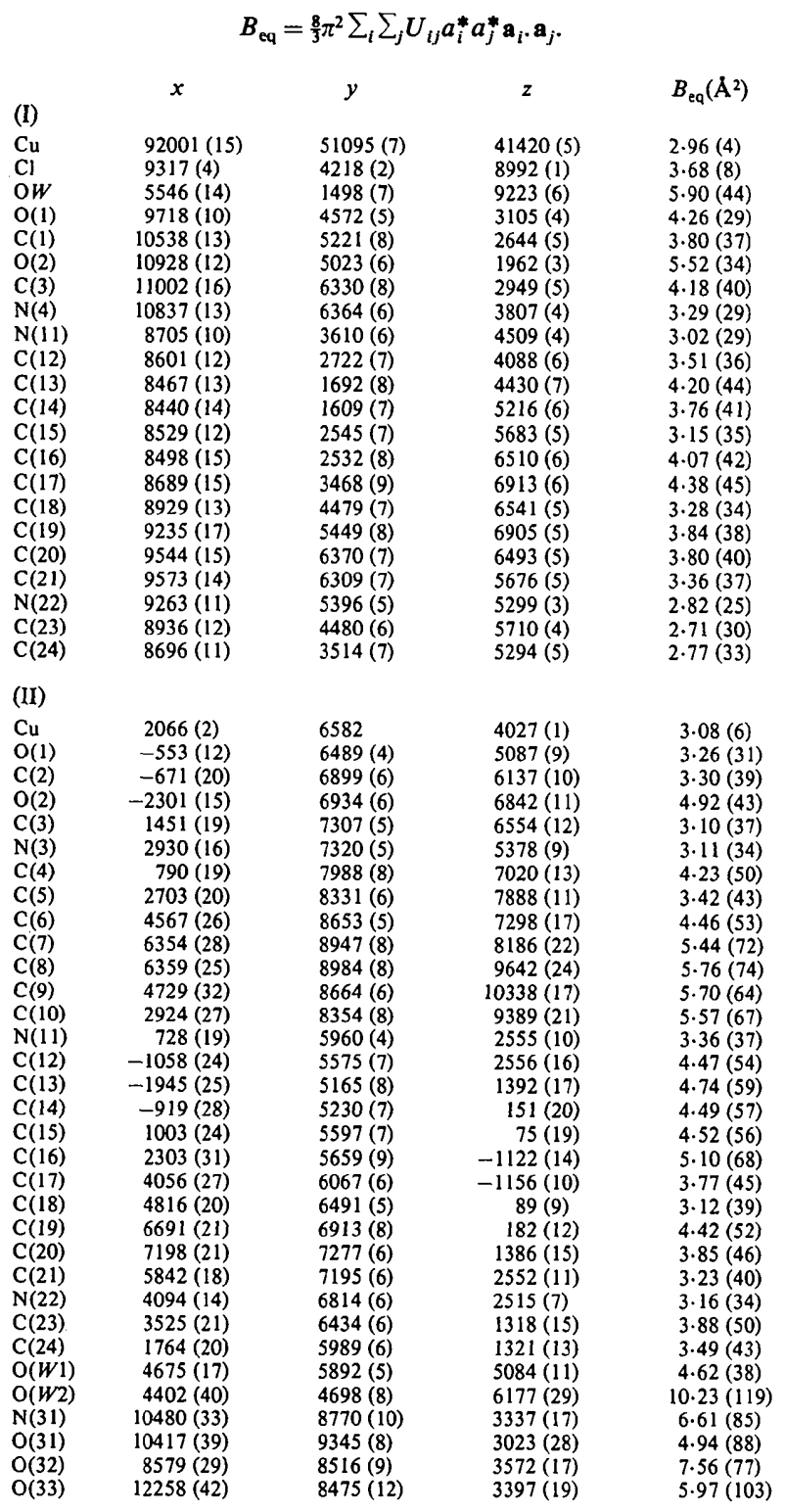

(I)

$\mathrm{Cl}-\mathrm{Cu}-2.546(2)$

$\mathrm{O}(1)-\mathrm{Cu} \quad 1.945(6)$

$\mathrm{N}(4)-\mathrm{Cu} \quad 2.007(7)$

$\mathrm{N}(11)-\mathrm{Cu} \quad 2.007(7)$

$\mathrm{N}(22)-\mathrm{Cu} \quad 2.031(6)$

$\mathrm{C}(1)-\mathrm{O}(1) \quad 1.265(11)$

$\mathrm{O}(2)-\mathrm{C}(1) \quad 1.233(10)$

$\mathrm{C}(3)-\mathrm{C}(1) \quad 1.516(13)$

$\mathrm{N}(4)-\mathrm{C}(3)-1.487(10)$

$\mathrm{C}(12)-\mathrm{N}(11) \quad 1.329(11)$

$\begin{array}{ll}\mathrm{C}(24)-\mathrm{N}(11) & 1.361(10)\end{array}$

$\mathrm{C}(14)-\mathrm{C}(13) \quad 1.361(15)$

$\mathrm{O}(1)-\mathrm{Cu}-\mathrm{Cl} \quad 100.9(2)$

$\mathrm{N}(4)-\mathrm{Cu}-\mathrm{Cl} \quad 103.4(2)$

$\mathrm{N}(4)-\mathrm{Cu}-\mathrm{O}(\mathrm{l}) \quad 84.5(3)$

$\mathrm{N}(11)-\mathrm{Cu}-\mathrm{Cl} \quad 100.6(2)$

$\mathrm{N}(11)-\mathrm{Cu}-\mathrm{O}(1) \quad 89.9(3)$

$\mathrm{N}(11)-\mathrm{Cu}-\mathrm{N}(4) \quad 156.0(3)$

$\mathrm{N}(22)-\mathrm{Cu}-\mathrm{Cl} \quad 93.5(2)$

$\mathrm{N}(22)-\mathrm{Cu}-\mathrm{O}(1) \quad 164.4(3)$

$\mathrm{N}(22)-\mathrm{Cu}-\mathrm{N}(4) \quad 97.7(3)$

$\mathrm{N}(22)-\mathrm{Cu}-\mathrm{N}(11) \quad 81.8(3)$

$\mathrm{C}(1)-\mathrm{O}(1)-\mathrm{Cu} \quad 116.0(6)$

$\mathrm{O}(2)-\mathrm{C}(1)-\mathrm{O}(\mathrm{I}) \quad 124.6(9)$

$\mathrm{C}(3)-\mathrm{C}(1)-\mathrm{O}(1) \quad 117.4(7)$

$\mathrm{C}(3)-\mathrm{C}(1)-\mathrm{O}(2) \quad 118.0(9)$

$\mathrm{N}(4)-\mathrm{C}(3)-\mathrm{C}(1) \quad 110.8(7)$

$\mathrm{C}(3)-\mathrm{N}(4)-\mathrm{Cu} \quad 107.9(6)$

$\mathrm{C}(12)-\mathrm{N}(11)-\mathrm{Cu} \quad 128.0(6)$

$\mathrm{C}(24)-\mathrm{N}(11)-\mathrm{Cu} \quad 113.4(5)$

$\mathrm{C}(24)-\mathrm{N}(11)-\mathrm{C}(12) \quad 118.1(7)$

$\mathrm{C}(13)-\mathrm{C}(12)-\mathrm{N}(11) \quad 122.2(9)$

$\mathrm{C}(14)-\mathrm{C}(13)-\mathrm{C}(12)$

119.1 (10)

(II)

$\begin{array}{ll}\mathrm{O}(1)-\mathrm{Cu} & 1.927(6) \\ \mathrm{N}(3)-\mathrm{Cu} & 2.004(9) \\ \mathrm{N}(11)-\mathrm{Cu} & 1.968(9) \\ \mathrm{N}(22)-\mathrm{Cu} & 2.010(8) \\ \mathrm{O}(W 1)-\mathrm{Cu} & 2.213(10) \\ \mathrm{C}(2)-\mathrm{O}(1) & 1.306(15) \\ \mathrm{O}(2)-\mathrm{C}(2) & 1.222(16) \\ \mathrm{C}(3)-\mathrm{C}(2) & 1.498(14) \\ \mathrm{N}(3)-\mathrm{C}(3) & 1.480(12) \\ \mathrm{C}(4)-\mathrm{C}(3) & 1.538(17) \\ \mathrm{C}(5)-\mathrm{C}(4) & 1.467(17) \\ \mathrm{C}(6)-\mathrm{C}(5) & 1.438(18) \\ \mathrm{C}(10)-\mathrm{C}(5) & 1.393(21) \\ \mathrm{C}(7)-\mathrm{C}(6) & 1.378(22) \\ \mathrm{C}(8)-\mathrm{C}(7) & 1.364(29) \\ \mathrm{C}(9)-\mathrm{C}(8) & 1.383(27) \\ \mathrm{C}(10)-\mathrm{C}(9) & 1.430(20) \\ \mathrm{C}(12)-\mathrm{N}(11) & 1.304(17)\end{array}$

$\mathrm{N}(3)-\mathrm{Cu}-\mathrm{O}(1) \quad 84.2(3)$

$\mathrm{N}(11)-\mathrm{Cu}-\mathrm{O}(1) \quad 91.8(4)$

$\mathrm{N}(11) \mathrm{Cu}-\mathrm{N}(3) \quad 169.4(4)$

$\mathrm{N}(22)-\mathrm{Cu}-\mathrm{O}(1) \quad 163.1(3)$

$\mathrm{N}(22)-\mathrm{Cu}-\mathrm{N}(3) \quad 98.2(4)$

$\mathrm{N}(22)-\mathrm{Cu}-\mathrm{N}(1 \mathrm{I}) \quad 82.9(4)$

$\mathrm{O}(W 1)-\mathrm{Cu}-\mathrm{O}(1) \quad 103.9(3)$

$\mathrm{O}(W 1)-\mathrm{Cu}-\mathrm{N}(3) \quad 96.4(4)$

$\mathrm{O}(W 1)-\mathrm{Cu}-\mathrm{N}(11) \quad 94.1(4)$

$\mathrm{O}(W 1)-\mathrm{Cu}-\mathrm{N}(22) \quad 92.4(3)$

$\mathrm{C}(2)-\mathrm{O}(1)-\mathrm{Cu} \quad 116.4(6)$

$\mathrm{O}(2)-\mathrm{C}(2)-\mathrm{O}(\mathrm{1}) \quad 124.4(10)$

$\mathrm{C}(3)-\mathrm{C}(2)-\mathrm{O}(1) \quad 116.1(10)$

$\mathrm{C}(3)-\mathrm{C}(2)-\mathrm{O}(2) \quad 119.3(11)$

$\mathrm{N}(3)-\mathrm{C}(3)-\mathrm{C}(2) \quad 110.5(9)$

$\mathrm{C}(4)-\mathrm{C}(3)-\mathrm{C}(2) \quad 111.4(9)$

$\mathrm{C}(4)-\mathrm{C}(3)-\mathrm{N}(3) \quad 112.5(8)$

$\mathrm{C}(3)-\mathrm{N}(3)-\mathrm{Cu} \quad 109.6(6)$

C(5)-C(4)-C(3) $\quad 113.8(9)$

$\mathrm{C}(6)-\mathrm{C}(5)-\mathrm{C}(4) \quad 124.1(10)$

$\mathrm{C}(10)-\mathrm{C}(5)-\mathrm{C}(4) \quad 122.5(13)$

$\mathrm{C}(10)-\mathrm{C}(5)-\mathrm{C}(6) \quad 113.4(13)$

$\mathrm{C}(7)-\mathrm{C}(6)-\mathrm{C}(5) \quad 120.9(15)$

$\mathrm{C}(8)-\mathrm{C}(7)-\mathrm{C}(6) \quad 122.0(16)$

$\mathrm{C}(9)-\mathrm{C}(8)-\mathrm{C}(7) \quad 122.1(14)$

$\mathrm{C}(10)-\mathrm{C}(9)-\mathrm{C}(8) \quad 114.2(16)$

$\mathrm{C}(9)-\mathrm{C}(10)-\mathrm{C}(5) \quad 127.1(18)$

$\mathrm{C}(12)-\mathrm{N}(11)-\mathrm{Cu} \quad 129.6(9)$

$\begin{array}{ll}C(15)-C(14) & 1.422(13) \\ C(16)-C(15) & 1.429(14) \\ C(24)-C(15) & 1.389(11) \\ C(17)-C(16) & 1.367(14) \\ C(18)-C(17) & 1.427(12) \\ C(19)-C(18) & 1.381(12) \\ C(23)-C(18) & 1.437(11) \\ C(20)-C(19) & 1.369(13) \\ C(21)-C(20) & 1.415(12) \\ N(22)-C(21) & 1.330(10) \\ C(23)-N(22) & 1.365(9) \\ C(24)-C(23) & 1.414(11) \\ & \\ & \\ C(15)-C(14)-C(13) & 120.1(9) \\ C(16)-C(15)-C(14) & 123.9(8) \\ C(24)-C(15)-C(14) & 116.5(8) \\ C(24)-C(15)-C(16) & 119.6(8) \\ C(17)-C(16)-C(15) & 119.9(8) \\ C(18)-C(17)-C(16) & 122.6(9) \\ C(19)-C(18)-C(17) & 126.1(8) \\ C(23)-C(18)-C(17) & 116.8(8) \\ C(23)-C(18)-C(19) & 117.0(7) \\ C(20)-C(19)-C(18) & 121.6(8) \\ C(21)-C(20)-C(19) & 118.4(8) \\ N(22)-C(21)-C(20) & 122.2(8) \\ C(21)-N(22)-C u & 129.5(5) \\ C(23)-N(22)-C u & 111.1(5) \\ C(23)-N(22)-C(21) & 119.4(6) \\ N(22)-C(23)-C(18) & 121.4(7) \\ C(24)-C(23)-C(18) & 120.4(7) \\ C(24)-C(23)-N(22) & 118.1(6) \\ C(15)-C(24)-N(11) & 124.0(8) \\ C(23)-C(24)-N(11) & 115.5(7) \\ C(23)-C(24)-C(15) & 120.5(7) \\ & \end{array}$

$\begin{array}{ll}\mathrm{C}(24)-\mathrm{N}(11) & 1.370(17) \\ \mathrm{C}(13)-\mathrm{C}(12) & 1.422(18) \\ \mathrm{C}(14)-\mathrm{C}(13) & 1.378(26) \\ \mathrm{C}(15)-\mathrm{C}(14) & 1.356(21) \\ \mathrm{C}(16)-\mathrm{C}(15) & 1.435(2) \\ \mathrm{C}(24)-\mathrm{C}(15) & 1.439(17) \\ \mathrm{C}(17)-\mathrm{C}(16) & 1.322(24) \\ \mathrm{C}(18)-\mathrm{C}(17) & 1.478(15) \\ \mathrm{C}(19)-\mathrm{C}(18) & 1.386(20) \\ \mathrm{C}(23)-\mathrm{C}(18) & 1.456(14) \\ \mathrm{C}(20)-\mathrm{C}(19) & 1.356(20) \\ \mathrm{C}(21)-\mathrm{C}(20) & 1.435(16) \\ \mathrm{N}(22)-\mathrm{C}(21) & 1.279(16) \\ \mathrm{C}(23)-\mathrm{N}(22) & 1.372(16) \\ \mathrm{C}(24)-\mathrm{C}(23) & 1.374(18) \\ \mathrm{O}(31)-\mathrm{N}(31) & 1.224(28) \\ \mathrm{O}(32)-\mathrm{N}(31) & 1.264(21) \\ \mathrm{O}(33)-\mathrm{N}(31) & 1.190(27)\end{array}$

$\mathrm{C}(24)-\mathrm{N}(11)-\mathrm{Cu} \quad 112.7(8)$ C(24)-N(11)-C(12) $\quad 117.5(10)$ C(13)-C(12)-N(11) 124.7 (14) $\mathrm{C}(14)-\mathrm{C}(13)-\mathrm{C}(12) \quad 115.8(13)$ $\mathrm{C}(15)-\mathrm{C}(14)-\mathrm{C}(13) \quad 123.1$ (14) $\mathrm{C}(16)-\mathrm{C}(15)-\mathrm{C}(14) \quad 127.4(15)$ C(24)-C (15)-C(14) 116.0 (15) C(24)-C(15)-C(16) $116.5(14)$ $C(17)-C(16)-C(15) \quad 123.9(14)$ C(18)-C(17)-C(16) $120.6(11)$ $\mathrm{C}(19)-\mathrm{C}(18)-\mathrm{C}(17) \quad 124.9(9)$ $\mathrm{C}(23)-\mathrm{C}(18)-\mathrm{C}(17) \quad 116.4(11)$ $\mathrm{C}(23)-\mathrm{C}(18)-\mathrm{C}(19) \quad 118.6(10)$ $\mathrm{C}(20)-\mathrm{C}(19)-\mathrm{C}(18) \quad 119.0$ (9) $\mathrm{C}(21)-\mathrm{C}(20)-\mathrm{C}(19) \quad 119.1(10)$ $\mathrm{N}(22)-\mathrm{C}(21)-\mathrm{C}(20) \quad 124.1(10)$ $\mathrm{C}(21)-\mathrm{N}(22)-\mathrm{Cu} \quad 131.3(7)$ $\mathrm{C}(23)-\mathrm{N}(22)-\mathrm{C}(21) \quad 118.7(9)$ $\mathrm{N}(22)-\mathrm{C}(23)-\mathrm{C}(18) \quad 120.3(10)$ $\mathrm{C}(24)-\mathrm{C}(23)-\mathrm{C}(18) \quad 120.9(11)$ $\mathrm{C}(24)-\mathrm{C}(23)-\mathrm{N}(22) \quad 118.8(10)$ $\mathrm{C}(15)-\mathrm{C}(24)-\mathrm{N}(11) \quad 122.5(12)$ $\mathrm{C}(23)-\mathrm{C}(24)-\mathrm{N}(\mathrm{II}) \quad 115.9(9)$ $\mathrm{C}(23)-\mathrm{C}(24)-\mathrm{C}(15) \quad 121.5(12)$ $\mathrm{O}(32)-\mathrm{N}(31)-\mathrm{O}(31) \quad 116.6(22)$ $\mathrm{O}(33)-\mathrm{N}(31)-\mathrm{O}(31) \quad 120.5(20)$ $\mathrm{O}(33)-\mathrm{N}(31)-\mathrm{O}(32) \quad 122.9(22)$ membered rings are planar [largest deviation 
$0.016(7) \AA$ at $\mathrm{C}(23)$ of (I)]. The $\mathrm{Cu}-\mathrm{O}-\mathrm{C}-\mathrm{C}-$ $\mathrm{N}$ (amino acid) five-membered chelate rings have an envelope conformation with N(4) 0.326 (7) $\AA$ out of plane in (I) and C(3) 0.256 (9) $\AA$ out of plane in (II). These variations in conformation are assigned to packing forces. In compound (I) $\mathrm{O}(2)$ and $\mathrm{N}(4)$ are hydrogen bonded to the hydrate water molecule [lengths: $\mathrm{O} W \cdots \mathrm{O}\left(2^{\mathrm{i}}\right) \quad 2 \cdot 805(8)$ and $\mathrm{O} W \cdots \mathrm{N}\left(4^{\mathrm{ii}}\right)$ $3 \cdot 15$ (2) Å; symmetry code: (i) $x-\frac{1}{2}, \frac{1}{2}-y, 1-z$; (ii) $\frac{3}{2} x$, $1-y, \frac{1}{2}+z$, while in (II) only $\mathrm{O}(W 1)$ (apical position) is strongly hydrogen bonded to $\mathrm{O}(W 2)\left[\mathrm{O}(W 2) \ldots \mathrm{O}\left(W 1^{\mathrm{i}}\right)\right.$ 2.69 (1) $\AA$; (i) $x, y, z]$.

This work was supported in part by the National Institutes of Health (USA) under Grant GM-19666 (REM). Two of us (LR and AM) thank the Facultad de Quimica UNAM-CONACYT (México) and the CSIC (Spain) for financial support.

\section{References}

Boys, D., Escobar, C. \& Martinez-Carrera, S. (1981). Acta Cryst. B37, 351-355.
Desiraju, G. R., Luss, H. R. \& SMith, D. L. (1978). J. Am. Chem. Soc. 100, 6375-6380.

GrIESSER, T. \& Sigel, H. (1970). Inorg. Chem. 9, 1238-1253.

International Tables for X-ray Crystallography (1974). Vol. IV, pp. 99-100, 149. Birmingham: Kynoch Press. (Present distributor D. Reidel, Dordrecht.)

Main, P., Fiske, S. L., Hull, S. E., Lessinger, L., Germain, G., DeclercQ, J.-P. \& Woolfson, M. M. (1980). MULTAN. A System of Computer Programs for the Automatic Solution of Crystal Structures from $X$-ray Diffraction Data. Univs. of York, England, and Louvain, Belgium.

Mégnamisi-Bélombé, M. \& Endres, H. (1983). Acta Cryst. C39, 707-709.

Nicholson, G. A., Petersen, J. L. \& McCormick, B. J. (1982). Inorg. Chem. 21, 3274-3278.

Phelps, D. W., Goodman, W. H. \& Hodgson, D. J. (1976). Inorg. Chem. 15, 2266-2271.

Rojo, T., Vlasse, M. \& Beltran-Porter, D. (1983). Acta Cryst. C39, 194-198.

Ruiz-Ramírez, L., Martínez, A. \& Gasque, L. (1988). In preparation.

SHELDRICK, G. M. (1976). SHELX76. Program for crystal structure determination. Univ. of Cambridge, England.

Solans, X., Ruiz-Ramírez, L., Gasque, L. \& Briansó, J. L. (1987). Acta Cryst. C43, 428-430.

Svedung, D. H. (1969). Acta Chem. Scand. 23, 2865-2871.

Van Meerssche, M., Germain, G., Declerce, J.-P. \& WilputteSteInerT, L. (1981). Cryst. Struct. Commun. 10, 47-51.

Acta Cryst. (1988). C44, 631-633

\title{
Structure of $\left\{N\right.$-[2-(2-Aminoethylamino)ethyl]salicylideneaminato- $\left.O, N, N^{\prime}, N^{\prime \prime}\right\}$ nickel(II) Perchlorate
}

\author{
By J. PodlahovÁ, K. KNížEK ANd J. LouB* \\ Department of Inorganic Chemistry, Charles University, Hlavova 8/2030, 12840 Praha 2, Czechoslovakia \\ AND J. HAŠEK \\ Institute of Macromolecular Chemistry, Czechoslovak Academy of Sciences, Heyrovského nám. 2 , \\ 16206 Praha 6, Czechoslovakia
}

(Received 25 September 1987; accepted 4 January 1988)

\begin{abstract}
Ni}\left(\mathrm{C}_{11} \mathrm{H}_{16} \mathrm{~N}_{3} \mathrm{O}\right)\right] \mathrm{ClO}_{4}, M_{r}=364 \cdot 43$, monoclinic, $\quad P 2_{1} / c, \quad a=8.930(4), \quad b=13.391(5), \quad c=$ 11.902 (6) $\AA, \beta=98.34(4)^{\circ}, V=1408$ (1) $\AA^{3}, Z=4$, $D_{m}=1.74(2), \quad D_{x}=1.718(1) \mathrm{Mg} \mathrm{m}^{-3}, \quad \lambda(\mathrm{Mo} K \alpha)=$ $0.71073 \AA, \mu=1.59 \mathrm{~mm}^{-1}, F(000)=752, T=295 \mathrm{~K}$, $R=0.078$ for 2498 unique observed reflections. The structure consists of $\left[\mathrm{Ni}\left(\mathrm{C}_{11} \mathrm{H}_{16} \mathrm{~N}_{3} \mathrm{O}\right)\right]^{+}$complex cations and perchlorate anions. The coordination polyhedron around $\mathrm{Ni}$ is a distorted square with the tetradentate $\mathrm{C}_{11} \mathrm{H}_{16} \mathrm{~N}_{3} \mathrm{O}^{-}$ligand (saden) bonded to the metal through one $\mathrm{O}$ and three $\mathrm{N}$ atoms. The perchlorate anion is disordered.
\end{abstract}

\footnotetext{
* To whom correspondence should be addressed.
}

0108-2701/88/040631-03\$03.00
Introduction. The study of the title compound was undertaken as part of an investigation of complexes with unsymmetrical tetradentate Schiff bases (Haber, 1986).

Experimental. The crystals were prepared by crystallization from a mixture of nickel(II) perchlorate, salicylaldehyde and diethylenetriamine from aqueous ethanol (Haber, 1986). Orange crystals were obtained, stable in air and to X-rays. Density determined pycnometrically. A sphere-shaped crystal of $r=$ $0.15 \mathrm{~mm}$ was used for the measurements. Syntex $P 2_{1}$ diffractometer, graphite monochromator, 20 reflections with $2 \theta$ from 8.86 to $20.66^{\circ}$ for measuring lattice (C) 1988 International Union of Crystallography 